\title{
ESTAR CERCA DE EUROPA. DEPORTE, CLASE SOCIAL Y PRESTIGIO EN ARGENTINA
}

\section{BEING CLOSE TO EUROPE. SPORT, SOCIAL CLASS AND PRESTIGE IN ARGENTINA}

\author{
Juan Bautista Branz ${ }^{1}$ \\ juanbab@yahoo.com.ar
}

Fecha de recepción: 8 de julio de 2015 - Fecha de aceptación: 30 de junio de 2016

\begin{abstract}
Resumen
El siguiente artículo propone pensar la clase social vinculada al deporte como espacio de sociabilidad y de distinción en Argentina. Mediante la idea de que las clases sociales se constituyen y se piensan, analíticamente, en movimiento. Además, se indaga cómo sujetos que practican rugby, en La Plata, otorgan sentido a su posición social, económica, política y cultural en relación directa al deporte recreado colectivamente por sectores dominantes en la ciudad. El rugby, en Argentina, es un deporte históricamente concebido y practicado por grupos minoritarios, no es una actividad de participación masiva. Se analizará cómo las influencias sociales y culturales que, desde Francia e Inglaterra, modelaron -y modelan- una forma particular de percibir el mundo entre hombres que juegan rugby en Argentina, excede al campo deportivo. El desafío de este trabajo es comprender la dinámica de estos espacios, desde la dimensión cultural de la clase social, en tanto experiencia que organiza las prácticas, ampliando la restringida definición económica.
\end{abstract}

Palabras clave: Rugby, Sectores dominantes, Distinción, Sociabilidad, Legitimidad

\begin{abstract}
The following article suggests thinking about social class linked to sport as a space of sociability and distinction in Argentina. Through the idea that social classes are constituted and are thought, analytically, in motion, it is investigated how subjects practicing rugby, in La Plata, give meaning to their social, economic, political and cultural position in direct relation to sport recreated collectively by dominant sectors in the city. Rugby in Argentina, is a sport historically conceived and practiced by minority groups, it is not an activity of mass participation. The analysis investigates how social and cultural influences from France and England, patterned-and pattern-a particular way of perceiving the world among men who play rugby in Argentina, exceeds the athletic field. The challenge of this work is to understand the dynamics of these spaces, from the cultural dimension of social class, while organizing practical experience, expanding the restricted economic definition.
\end{abstract}

Keywords: Rugby, Dominant sectors, Distinction, Sociability, Legitimacy

1 Conicet/Ideas, FPyCS/UNLP, Argentina. 


\section{Introducción}

En el primer apartado de este escrito se presentan las técnicas e instrumentos utilizados para construir los datos que se exponen en los siguientes pasajes ${ }^{2}$.

El segundo tramo describe las características de la ciudad de La Plata, donde se realizó la investigación que permite formular discusiones en torno a relaciones entre deporte, clase y prestigio.

El rugby, en la ciudad de La Plata, y en Argentina, es un espacio donde se reproducen tradiciones vinculadas a los grupos que, históricamente, modelaron y obtuvieron legitimidad y autoridad cultural para ordenar el sentido de ciertas prácticas sociales. El rugby, traduce y cristaliza un 'sentido europeo', como matriz sociocultural de pensar, percibir y actuar en el mundo.

A continuación, se conceptualiza la noción de clase social en/desde el campo de las prácticas deportivas que desarrollan hombres de sectores dominantes. Se repasa la articulación de las experiencias francesas e inglesas, en tanto antecedentes que marcaron a los grupos aristocráticos que se apropiaron del rugby en Argentina -logrando distinguirse de otros espacios sociales- y de una cultura masiva como manera de despegarse material y simbólicamente de actividades vinculadas con sectores medios trabajadores y sectores populares.

Finalmente, se revisa la idea de estar cerca de Europa como signo distintivo de los sectores dominantes en Argentina y como modo de concebir qué es lo legítimo entre las prácticas culturales y sociales, superando la determinación de las condiciones económicas.

\section{Técnicas e instrumentos}

Desde finales del 2009, y durante los años siguientes, hasta 2013, el programa metodológico apuntó a trabajar las variables a partir de las técnicas pertinentes:

- Etnografía. Permitió construir datos sobre las relaciones entre sujetos, espacios y discursos.

- Treinta y cinco entrevistas semi-estructuradas a jugadores de rugby. La desagregación de trayectorias familiares e historias de vida, permitieron ubicar y categorizar, provisoriamente, a los interlocutores en la estructura socioeconómica, y a la posición vinculada a la distribución de capitales en juego en nuestras sociedades. Con los datos se construyeron categorías como: profesión, participación en el sistema educativo, titulaciones, profesión de padre, madre y familiares. Así se vislumbró la tendencia de los participantes del campo: cuántos sujetos de la investigación han accedido al sistema de educación universitaria, o a cargos de decisión en la órbita del Estado, o del sector privado. Esto facilitó pensar el problema de los sectores dominantes y el espacio social, en una de sus dimensiones.

- Ochenta y nueve entrevistas etnográficas a diferentes agentes del campo (familiares de jugadores, dirigentes, periodistas especializados, espectadores, ex jugadores): permitieron comprender la formación discursiva.

- Observación participante en entrenamientos, practicando el deporte y desarrollando la experiencia propia del "hacer" y la observación no participante de partidos, en un gimnasio de musculación (coordinado por uno de los interlocutores clave), en fiestas nocturnas, cumpleaños, espectáculos artísticos, salidas nocturnas, peñas folklórica ${ }^{3}$, viajes de ocio, partidos oficiales, trámites varios,

2 Este texto se desprende de la Tesis Doctoral realizada en el marco del Doctorado en Comunicación,(Universidad Nacional de La Plata, 2015), titulada "Deporte y masculinidades entre sectores dominantes de la ciudad de La Plata. Estudio sobre Identidades, Género y Clase”, dirigida por el Dr. José Garriga Zucal y Co-dirigida por el Dr. Pablo Alabarces.

3 Una peña folklórica es un espacio de encuentro, de sociabilidad, donde se escucha y se baila música reconocida como regional, propia de géneros y estilos argentinos. 
situaciones domésticas familiares y "tercer tiempo" . Un total de 223 presencias en los diferentes espacios nombrados durante los cinco años de trabajo de campo.

- La búsqueda y recopilación de documentos de campo, vinculados a la historia del rugby en Argentina, en La Plata, y en los clubes, enmarcaron historiográficamente la investigación, y colaboraron al entendimiento actual de las lógicas del campo.

- Búsqueda e interpretación de documentos periodísticos especializados en rugby, audiovisuales y gráficos.

\section{La Plata: ciudad "mágica"}

Es la ciudad capital de la provincia de Buenos Aires ${ }^{5}$, situada a 50 kilómetros de la Capital Federal, Ciudad Autónoma de Buenos Aires. Tiene una población estimada en 700.000 habitantes distribuidos en una superficie de 926 kilómetros cuadrados. Sus actividades principales son la administración estatal, el comercio y la educación ya que la Universidad Nacional es una institución de prestigio que convoca a miles de estudiantes del país y de Latinoamérica.

Resulta curiosa la historia de su fundación, ya que es una ciudad que tomó "vida" desde una idea ambiciosa: el proyecto político que diseñó, modeló y organizó la nueva Capital de la provincia de Buenos Aires.

El principal organizador de la ciudad fue el gobierno bonaerense que, a través de la creación de un Estado, debió instalar no solamente las instituciones sino "inventar" y seducir a su población. La arquitectura grandiosa, los avances tecnológicos, o las facilidades para la radicación no resultaron suficientes para concretar una ciudad "habitada": resultaba una ciudad fastuosa, pero desierta; sin sujetos que la doten de pertenencia ni identidad. Identidad soñada y configurada sobre los valores constitutivos del proyecto sustentados en los postulados de la Generación del '80: la razón iluminista, la ciencia, el progreso, la felicidad, el orden, la belleza.

La población se fue asentando a partir de créditos para vivienda otorgados a los obreros y técnicos que participaron en la construcción de los edificios gubernamentales. La aplicación de la Ley de Residencia ${ }^{6}$ implicó la instalación en la ciudad, estableciendo domicilio permanente. Para facilitar ese proceso se impulsó la distribución de tierras fiscales a funcionarios y empleados que debían viajar ida y vuelta a la ciudad de Buenos Aires. Los modos de repartir y privatizar las tierras marcaron fuertemente las particularidades del negocio inmobiliario.

Estos primeros habitantes fueron imprimiendo sus trayectorias tan distantes como diversas: contingentes migratorios europeos convivieron con miembros de familias patricias porteñas. Estas divergencias, cuya base se fundamentaba en el modelo del "progreso", condenaron a la desidia a miembros de pueblos originarios, a campesinos, a gauchos y pastores.

A partir de la radicación de estos sectores se desarrollaron, muy rápidamente, múltiples actividades que gestaron y articularon campos especializados en la política, la educación, la salud, la alimentación, la cultura, los servicios, el ocio, el transporte, el deporte, la construcción y las finanzas.

4 El "tercer tiempo" es realizado luego del partido de competencia. Históricamente, como ritual, el equipo local recibe a su rival con un agasajo que consiste en compartir desde bebidas como té, hasta alcohólicas, acompañadas de algún alimento dulce y/o salado. En ese espacio, ampliamos el espectro de actores conocidos dentro de los clubes.

5 Fuente: Ministerio del Interior, Obras Públicas y Vivienda. Presidencia de la República Argentina.

6 La Ley de Residencia fue promulgada por el Congreso de la Nación Argentina en 1902. Permitía que el Estado argentino expulsara a inmigrantes sin que estos pudieran apelar su exclusión del país. La ley fue impulsada por el escritor Miguel Cané y fue derogada en el año 1958. También se conoce como la Ley 4.144 de Residencia. 
Cada campo fue fundando tradiciones a través de instituciones, agentes y prácticas que contribuyeron dinámicamente a poner en acto a "una leyenda" sobre la identidad platense.

Las tres unidades de observación construidas para iniciar el trabajo de campo fueron el Club Universitario de La Plata, La Plata Rugby Club (LPRC) y Albatros Rugby Club. Los dos primeros están ubicados en la localidad de Manuel Bernardo Gonnet, aproximadamente a 10 kilómetros al norte del centro de la ciudad de La Plata. Es una zona residencial, con una densidad poblacional baja, cuyos lotes (en gran parte del barrio) se caracterizan por sus grandes dimensiones y por su particularidad de "casasquintas”. El Club Albatros se sitúa en la localidad de José Hernández, a aproximadamente 30 kilómetros al noroeste del centro de la ciudad de La Plata. Se caracteriza por ser una zona residencial, con una densidad poblacional media/baja, donde confluyen tanto viviendas como comercios de media y gran escala.

\section{Para pensar la clase en la ciudad de La Plata: aproximaciones conceptuales}

A decir de Williams (2003), en Palabras Clave, una clase es en ciertas coyunturas una categoría económica, que contempla a quienes se encuentran objetivamente dentro de una situación económica determinada. Pero también, siguiendo a Marx, conceptualiza a la clase como una formación históricamente determinada, donde se desarrollan conciencias de situaciones y de organización para enfrentar la posición hostil en que puede colocar una clase a otra, en relación a los modos de vida, a los intereses y sus culturas.

Gerth y Wright Mills (1963) en Carácter y Estructura Social, ponen énfasis en el concepto de 'estratificación' para pensar las clasificaciones que organizan la reflexión sobre el orden social. Así, de acuerdo al criterio de clasificaciones donde se ponen en juego qué cosas y qué experiencias son valoradas por diferentes grupos sociales, se pueden delimitar las expectativas que la gente tiene regularmente, los valores que circulan, y los modos de esa circulación. Esto determina la posibilidad de pensar en la estratificación social como criterio analítico.

Gerth y Wright Mills encuentran en su estudio cuatro claves del fenómeno de las sociedades estratificadas que llamarán "dimensiones de la estratificación”. Estas son: la ocupación, la clase, el estatus y el poder. La ocupación entendida como la acumulación de las actividades realizadas como fuente de ingresos. La situación de clase, en relación a la cantidad y la fuente de ingresos (que puede traducirse en propiedades o trabajo), dispuestas a otorgar a la gente las posibilidades de conseguir otros valores que circulan.

El estatus como el resultado directo entre el cumplimiento eficaz de las expectativas en relación a obtener prestigio social; la referencia que hacen Gerth y Wright Mills, es directa a la distribución del respeto en una sociedad. Y el poder, como ejecución de la voluntad de uno, aún si ésta depende de la intransigencia de otros. Aclaran Gerth y Wright Mills, que la conceptualización en torno al estatus, también se aplica al poder. Es decir, comprende todos los roles instituidos en diferentes campos (económico, religioso, familiar, político) que impliquen relaciones de autoridad. Por eso, el estatus dependerá de la diversidad de roles que una persona asuma, según sean las instituciones en las que participe y el grado de poder que administre en cada una de ellas. En términos de roles, definen al rol económico como parte de lo que denominan ocupaciones, determinadas a su vez por el conjunto de actividades destinadas a la subsistencia material y orientadas a un mercado de trabajo que proporciona el intercambio de bienes y servicios.

Los criterios de clasificación socioeconómica y la conceptualización de ocupación resultaron relevantes, ya que permitieron marcar una cierta regularidad y un vínculo estable entre lo que los sujetos hacen para vivir. Es lo que Gerth y Wright Mills nombraran como "rutina", y cuando esa "rutina" se hace para obtener un ingreso regular. De ahí, la preocupación en agrupar a los hombres investigados, según su ocupación.

De esa manera, se estableció una relación entre la posición de clase y estatus. Se insiste, nuevamente, en rastrear la ocupación de los hombres que juegan al rugby en la ciudad de La Plata, aunque la 
intención fue pensar cómo se produce, se percibe y se distribuye el prestigio, no tanto entre sus esferas de trabajo, sino por fuera de ella: en el mismo campo del rugby, y en otros espacios del mundo social.

Ahora bien, pensando en combatir la visión instrumental y determinista de clase, Gerth y Wright Mills insisten en que las probabilidades de que una clase dé cuenta y se sirva de las posibilidades, dentro de una estructura de clases, no significa que los miembros de esa clase sean -necesariamente- conscientes de su posición, o de posiciones similares a otro grupo de personas. Piensan que no es indefectible que una clase se sentirá unida, ni que "dará cuenta de intereses que objetivamente similares se pueden atribuir a su condición como racionalmente convenientes. Tampoco necesita definir intereses similares como intereses comunes, y organizarse para alcanzarlos en un movimiento o partido" (Gerth y Wright Mills, 1963, p. 294).

Las anteriores consideraciones permitirían entender que las formaciones de clase no son consecuencia lógica e histórica del hecho objetivo de la estructura toda. En tal sentido, se podrían establecer regularidades en torno a trayectorias sociales, culturales, económicas y políticas; que muchas veces se presentan difusas, confirmando que el estudio y la conceptualización sobre las clases, conlleva más imperfecciones en la manera de asir y agrupar elementos que distingan a un grupo de personas de otras. Es por eso que el interés residió en abrir las preguntas hacia las heterogeneidades de los espacios sociales, para brindar más posibilidades de interrogar las dinámicas sociales, culturales y simbólicas, y entender los procesos desiguales en la repartición de los capitales que circulan.

Por otro lado, Thompson (1989) se vincula lo impreciso a la pregunta por la clase como una oportunidad, concibiendo a la clase como fenómeno histórico, como la sucesión de acontecimientos en apariencia sin conexión, tanto con lo que entiende como materia prima de la experiencia, como con la conciencia. En ese mismo sentido, rechaza la clase como "estructura" y, aún más como "categoría”, para pensarla como "algo que tiene lugar de hecho (y se puede demostrar que ha ocurrido) en las relaciones humanas" (Thompson, 1989, p. 13). Y reafirma que ni "el entramado sociológico mejor engarzado puede darnos una muestra pura de clase, del mismo modo que no puede dárnosla de la deferencia o del amor. La relación debe estar siempre encarnada en gente real y en un contexto real" (Ibíd.). La experiencia de clase está determinada, según se consignó anteriormente, y siguiendo a Thompson, por las relaciones de producción en la que los hombres nacen o entran involuntariamente. Puede surgir la conciencia de clase en algún momento y lugar, pero no de la misma forma para todos.

La conciencia de clase es "la forma en que se expresan estas experiencias en términos culturales: encarnadas en tradiciones, sistemas de valores, ideas y formas institucionales" (Thompson, 1989, p. 14). Se piensa a la clase como experiencia; de lo contrario, se caería en el mecanicismo que se intenta discutir y sortear para el análisis de este escrito: "Si recordamos que la clase es una relación, y no una cosa, no podemos pensar de este modo" (Ibíd., p.15)

Se adscribe, siguiendo la línea crítica, a la postura que retoma Hall (1994) cuando persigue la discusión que dio Raymond Williams contra las operaciones literales de la metáfora base/superestructura. Esto era caducar la creencia que sostenía el marxismo clásico, cuando asignaba las ideas y los significados a la "superestructura", como reflejo absoluto y determinado de la "base" material. Discutiría contra el materialismo vulgar y el determinismo economicista, poniendo el foco sobre lo que denominaría "formas de organización" que subyacen de las prácticas, definiendo a la cultura como modo de pensar las relaciones sociales de forma compleja. Hall dice de Williams que,

Define a la cultura como los significados y los valores que emergen entre grupos y clases sociales diferenciados, sobre la base de sus condiciones y relaciones históricas dadas, a través de las cuales 'manejan' y responden a las condiciones de existencia; y como las tradiciones y prácticas vividas a través de las cuales son expresadas esas 'comprensiones', y en las cuales están encarnadas (Hall, 1994, p. 34)

Hall, piensa las posiciones de Williams y Thompson, y sostiene que las dos leen las relaciones en términos de cómo son "vividas" y "experimentadas" (Hall, 1994, p. 34). Williams desde las "estructuras 
de sentimiento"7 y Thompson desde el carácter dado -históricamente- de las relaciones en el que los hombres y las mujeres ingresan involuntaria y necesariamente, bajo los signos de la explotación del sistema capitalista. La experiencia de los sujetos y el papel a desarrollar en esas relaciones, será central.

\section{Rugby y clase social}

Pensar contextual y relacionalmente es central en cualquier estudio que se pretenda riguroso. El acercamiento al rugby, como punto de partida para pensar problemas en torno a la clase y al género, necesitó de una mirada histórica y situacional. Las preguntas se enfocaron -todo el tiempo- en el porqué de construir al rugby como deporte vinculado a una distinción de clase. Se recorrió históricamente la relación que determina las características de una ciudad como La Plata, y la cultura que organiza alguna de las prácticas del colectivo sujeto de este estudio. Y también, se articuló -analíticamente- esa invención cultural urbana, con agentes, prácticas, instituciones y tradiciones vinculadas al rugby en la ciudad demostrando que, como mínimo, no es un deporte masivo, ni plural, en torno a quienes lo piensan, lo organizan y lo practican. El objetivo de este trabajo (entre otros) fue reponer el concepto de clase y de dominación, para pensar en la desigual distribución de capitales entre nuestras sociedades y, también, demostrar por qué los valores y las prácticas del mundo del rugby confirman y garantizan la reproducción de esa desigualdad que se extiende, por supuesto, más allá del rugby. Poner en tensión la teoría, con la referencia empírica construida, resultó todo un desafío.

\section{El rugby, Inglaterra y la reivindicación de las clases}

Tony Collins describe cómo las clases medias y clases trabajadoras, durante la época Victoriana en Inglaterra, se apropian del rugby (de su práctica, de su estilo), pensando en la exploración de los cambios culturales y las conquistas políticas, sobre todo, entre trabajadores del Norte. Pone en el centro de la discusión la noción de clase, en términos históricos, partiendo de usos y configuraciones de una práctica (el rugby) cuyo valor era determinado, en principio y exclusivamente, por estudiantes pertenecientes a las Public Schools.

En este contexto, y ante la expresión del tiempo libre, como tiempo delimitado fuera del horario ocupado por/para el trabajo, Collins analiza la relación entre las clases medias (las asociadas a las ocupaciones "de cuello blanco"),

More importantly for the broader perception of the sport, the myth served to anchor the Rugby game as separate from the older traditions of plebeian folk football, creating a distinct middle-class lineage for the sport at a time when the middle classes in general were seeking to create exclusive recreational havens for themselves outside of the prevailing mass sporting culture. (Collins, 2006, p.6)

[Aún más importante para una percepción más amplia del deporte, el mito sirvió para anclar al juego del rugby como algo distinto de las tradiciones más antiguas del fútbol popular plebeyo, creando un claro linaje de clase media del deporte al mismo tiempo que las clases medias en general procuraban crear exclusivos refugios recreativos distinto de lo que era la imperante cultura del deporte de la masa (Traducción propia)]

El rugby significaba la invención y recreación de un espacio distintivo para las clases medias. Propio de un colectivo y alejado de todo lo que implicara una cultura de masas. Es un momento clave: las tradiciones pre-capitalistas, asociadas a una cultura plebeya, deben ser despojadas y distinguidas de

7 En el escrito de Hall está referenciada la noción de "estructura de sentimiento" de Williams, como la relación que se establece entre las estructuras mentales. Como las categorías que organizan la conciencia empírica de un grupo determinado relativas a un mundo imaginario creado colectivamente. 
una nueva forma de vincularse, articulando valores que expresen esa distinción que, además de clase, era al mismo tiempo un distanciamiento que operaba en clave de género,

For example, at various points the relationship between rugby and masculinity is looked at in the context of public school rugby, under rugby union rules and Northern Union rules. I will argue that, first and foremost, definitions of masculinity and violence were defined by class. Acts perceived as manly and character-forming by the middle classes were interpreted differently when carried out by members of the working classes: thus hacking was viewed as courageous between former public school boys, yet outrageous when perpetrated by miners. Conversely, the predominantly workingclass supporters of the NU found tripping and kicking unacceptable. The growth of imperial nationalism towards the end of the nineteenth century had a crucial impact on notions of masculinity (Collins, 2006, p.18)

[Por ejemplo, en distintos puntos, la relación entre el rugby y la masculinidad se analiza en el contexto del rugby de las escuelas públicas, según las reglas de la asociación de rugby y también de aquellas de la Liga del Norte. Primero y principal, considero que las definiciones de masculinidad y de violencia se definían por clase. Los actos considerados masculinos y formadores de carácter de las clases medias eran interpretados de forma diferente cuando se llevaban a cabo por miembros de las clases trabajadoras: mientras que el pirateo era visto como un acto de valentía entre los primeros alumnos de las escuelas públicas, éste se convertía en atroz cuando era perpetrado por mineros. Por el contrario, los simpatizantes de la Liga del Norte, en su mayoría de la clase trabajadora, consideraban inaceptables los tropiezos y puntapiés. El crecimiento del nacionalismo imperial hacia fines del siglo XIX tuvo un fuerte impacto en las nociones de masculinidad (Traducción propia)]

Aquí radica la operación simbólica y su eficacia sobre la misma práctica al asociar el deporte a la clase social, y en asentar una definición y percepción legítima, según la clase, sobre la masculinidad y la conducta varonil justamente, según qué clase la definía y la percibía.

Pero más allá de esta diferenciación simbólica, la disputa de clases representada en el uso y en la configuración de la práctica, tanto de la clase trabajadora como de los dueños del capital, no llegaba a una confrontación absoluta. Collins advierte una posible reducción de las tensiones, en torno al significado otorgado por cada colectivo al rugby, al encontrarse ocasionalmente, trabajadores y dueños de fábricas en un mismo partido (como rivales o, frecuentemente, como compañeros de equipo). Esto indica que la apropiación por parte de la clase trabajadora tiene aún mayor importancia, más allá del intento de deslegitimar el uso de la práctica por parte de la clase dirigente; porque implica que la distinción no es totalmente excluyente. Hay un registro del "otro" como colectivo que se intenta negar, pero que termina siendo positivizado al compartir los mismos espacios. Lo que no implica igualdad en derechos ni en condiciones, ni materiales ni simbólicas. La disputa y la dominación seguían siendo efectivas y reales, como se puede observar en la siguiente cita:

This did not mean that class conflict had been abolished or that the leisure pursuits of the classes were interchangeable. Nor did it mean that harmonious relations between the classes were the norm. Practically, it meant that the reduction in social tensions created a greater possibility of members of different classes playing sports together, certainly when compared to the pre- and post- 1848 periods. In rugby, this process manifested itself in factory owners and factory workers playing the same game and occasionally in the same teams (Collins, 2006, p.24)

[Esto no quiere decir que la lucha de clases había sido abolida o que las actividades de ocio de las clases eran intercambiables tampoco significa que las relaciones armoniosas eran la norma entre las clases. En la práctica, eso significaba que la reducción de las tensiones sociales había creado más posibilidades para que miembros de diferentes clases estén haciendo deporte juntos, sin duda, en comparación los períodos pre -y post-1848. En el rugby, este proceso se manifestó entre los dueños de las fábricas y los trabajadores de las fábricas que juegan el mismo juego y de vez en cuando en los mismos equipos (Traducción propia]

La relación entre el tiempo dedicado a la práctica y la remuneración por practicar el deporte fue central para discutir la incorporación de la clase trabajadora al rugby, y definir las categorías de profesionales y amateurs, con los valores asociados a cada condición. La asignación de valores negativos 
hacia el profesionalismo operaba en detrimento de la clase obrera que recibía dinero por jugar. Allí se jugaba la operación de exclusión, al negativizar la práctica rentada. La distinción entre lo amateur y lo profesional era un recurso para excluir simbólicamente a quienes eran remunerados, aunque se encubriesen y solapasen las diferencias, no sólo del tiempo libre disponible entre las clases, sino del significado y del sentido de ese tiempo libre para las clases. Collins (2006), sostiene que aunque no fuese enunciado como tal, el fútbol rugby se había convertido en un punto de conflicto entre la expresión de las prácticas culturales de la clase trabajadora y los códigos culturales dominantes del carácter distintivo de la escuela pública.

Una relación de distanciamiento con necesidades materiales era lo que definía, según Collins, la posición de las clases medias y su vinculación a la práctica de rugby. Sin dudas, el rugby se había convertido en el vehículo de una disputa simbólica entre los significados y los usos de clase, sobre el tiempo libre y el ocio.

\section{Francia: del elitismo a la popularización}

Philip Dine (2007), revisa las inversiones ideológicas que se incluyen en el deporte en Francia, y reconstruye históricamente la relación específica entre la clase, el rugby y el cuerpo, como soporte de significaciones, de representaciones culturales, como "bóveda" de un edificio social donde ciertas clases encuentran beneficios morales, psicológicos e incluso patrióticos.

Concebido en el siglo XIX como el deporte distintivo de los niños de familias dirigentes inglesas, dice Dine que el rugby expresa sus virtudes masculinas tradicionales (coraje, resistencia, fuerza), inspirados en un "cristianismo muscular" en un marco educativo. Pero que lleva a constituir al rugby como la base de un deporte exclusivo de una clase exhibiendo valores dominantes como los asociados a la "patriarquía", el nacionalismo, el chovinismo y el militarismo, el colonialismo y el racismo. El cuerpo modelado en el rugby es masculino e inglés, imperialista y blanco. De aquí que la aceptación fuera consonante en los territorios británicos poblados en el hemisferio sur que cristalizan y conservan casi como "feudos" el deporte. Es que el rugby se había convertido en un vehículo de las virtudes primeras, expandidas entre los colonos australianos, neozelandeses o sudafricanos antes de permitir la expresión de nuevas identidades nacionales.

En una primera instancia, la expansión del rugby en Francia se dio entre sectores de las elites parisinas, aunque hubo un proceso de popularización, que Dine recupera, historiográficamente, para lo cual basa su análisis en la importancia de elementos como el sentimiento de regionalismo y las identidades locales. También es notorio el sentido homogeneizante de una identidad nacional.

A través del discurso higienista de la educación física, en algunas zonas del campesinado y del sector obrero, el rugby se convierte en un fuerte operador identitario, promoviéndose en la Liga Girondina, donde los éxitos del equipo Stade bordelais motivan hacia la práctica y amplía el número de participantes. El rugby se populariza y adquiere ciertos movimientos democratizantes en la década de 1920, siendo una práctica burguesa hasta 1914. El rugby es representado entre el campesinado francés como disposición típicamente popular y de culto a la virilidad y el gusto por el contacto cuerpo a cuerpo con dureza. Además de ser un espacio de encuentro y celebración (especialmente "el tercer tiempo") entre hombres tanto de la elite parisina, como de sectores trabajadores de Bordeaux o de grandes regiones del gran sudoeste francés.

Durante los años '30 (del siglo XX), el rugby francés se transforma en un espacio que representa conflictos sociales y políticos, desde el Frente Popular hasta el régimen de Vichy. La administración y la visibilidad que adquiere el rugby en sentido político e ideológico es único, y es codiciado por todos los movimientos políticos (Dine, 2007). Pero es el régimen de Vichy quien llega a hacer del rugby en el campesinado un pilar de su programa de "vuelta a la tierra", en el marco de su "Revolución nacional". Las inversiones morales y materiales de Vichy le permiten el éxito al rugby como práctica en el período de post-guerra, siendo adoptado por los gobiernos sucesivos como signo nuevo de eficacia (sobre todo, en una especie de reposición de la virilidad) en un país que intenta reconstruirse tras la segunda guerra mundial (Ibíd.) 


\section{Francia e Inglaterra: los modelos a seguir en Argentina}

En Argentina, la devoción por la cultura europea (especialmente, por lo británico y lo francés) por parte de los sectores dominantes y dirigentes se convirtió, en el campo del rugby, en la copia fiel de la práctica europea. Dice Alabarces (2002) que es en la década de 1910 que los equipos británicos de fútbol abandonan los torneos, para organizar y diseñar las tradiciones del rugby en Argentina. Con algunos puntos de contacto con la experiencia inglesa, las clases populares argentinas se reapropian del uso y la configuración de la práctica futbolística, cambiando su sentido cultural y social, en lugar del esquema ideológico y de clase inglés (Archetti, 2001 y Alabarces, 2002). El rugby y el hockey son, durante varios años, un fuerte símbolo de distinción de clase en Argentina (Alabarces, 2006).

La concepción de Fair Play guarda relación directa con una noción de honor, respeto y lealtad con el que los gentlemen ingleses se diferenciaban de aquellos que no podrían hacer del autocontrol de sus emociones una señal distintiva. Palermo (2010) exhibe cómo la apropiación del rugby por parte de los sectores dominantes, en la Argentina, a fines del siglo XIX y principios del siglo XX se sostuvo en una reinterpretación de "lo ingles" como elemento distintivo. Si los estudios de Archetti (2001) sobre las representaciones fundacionales de la Nación argentina (de un estilo de juego y de masculinidad) de la Revista El Gráfico (desde los años '20, en adelante) mostraron que desde el fútbol se construía un mundo imaginario donde lo masculino no se relaciona con la pureza del guerrero, con la virilidad y el sacrificio, como la experiencia francesa o inglesa, el mundo imaginario de los sectores dominantes se hará cargo de esos atributos y los asociará directamente al rugby como espacio significante: pero además le agregará el sentido de la caballerosidad, asociada históricamente al espacio de "lo inglés". Es la operación inversa desarrollada en la historia del fútbol en Argentina. Tal vez porque no haya un sujeto colectivo que disputó e interpeló con su participación al aristocrático rugby argentino. Se trata de la inversión ideológica que reproduce una forma moral de ser hombre y concentrar atributos como la fuerza, la caballerosidad y la elegancia, en un mismo cuerpo (Branz y Garriga, 2012).

Desde el rugby, históricamente, se concibió la oportunidad de conciliar en un mismo espacio, por un lado, la condición de caballerosidad (basadas en la tolerancia, la lealtad, el respeto y la disciplina) y, por otro, la agresividad (asociada a las características de violencia de la práctica). En apariencia, si se remite a la condición dualista, de lo dócil y lo agresivo, o lo violento y lo pacífico, se establece una oposición que, en el campo de rugby, se presenta como complementaria o necesaria. Es decir, el sistema elaborado históricamente en base a modelos civilizatorios que regularon el espacio del rugby en Argentina, como vínculo deportivo con los sectores dominantes, a través de una lógica apoyada en la razón como forma "descubrir" el mundo y construir las propias prácticas, estabilizó y garantizó la necesidad de resguardar un espacio distintivo de clase y, conjuntamente, un lugar seguro para los atributos asociados a una forma tradicional de masculinidad. Una producción y reproducción cultural vuelta naturaleza, vuelta sentido práctico (Bourdieu, 2007[1980]).

\section{Experiencias en campo ${ }^{8}$}

Con el tiempo Nacho ${ }^{9}$ amplió su criterio sobre la diferencia de los clubes (entre su Club y el resto de los indagados en la investigación), y al argumento socioeconómico sostenido por la geopolítica

8 En este apartado se cambiará el registro de escritura a la primera persona del singular y del plural, dado que se transcribirán apuntes y pasajes del diario de campo (observación), a partir del cual se construyeron los datos que responden a los objetivos centrales de la investigación. La idea de conservar el registro se entiende como un recurso para intentar mantener la fidelidad del lenguaje y de las prácticas de los sujetos investigados, para reconstruir su trama narrativa; emparentada, por supuesto, con el ejercicio reflexivo de la participación del investigador inmerso en el campo. Los entrecomillados indican la reproducción textual de lo nombrado por los sujetos investigados.

9 Nacho es Profesor de Educación Física, es propietario de un Gimnasio, y ha sido un interlocutor clave para mi trabajo. Juega en el Albatros Rugby Club y he compartido muchas horas y días con él y sus compañeros de equipo en ese gimnasio. 
territorial, le sumó un componente étnico/cultural. De la diferenciación general que, ubicaba en la periferia y posición subalterna -a su Club- con respecto a los demás clubes, Nacho redujo y recortó la caracterización (recurriendo, igualmente, a lo territorial como fundamento que organiza cada grupo). La distancia inicial con Universitario, disminuía si los "otros" distantes eran los habitantes de City Bell ${ }^{10}$; entonces la diferencia se vuelve relativa agregando otros componentes: "No hay diferencia, obviamente la diferencia está dada a que 'son los negros de Hernández' o ‘son los negros de Gonnet' o son 'los chetos de City Bell', para los de City Bell son 'los negros de Gonnet'.' La 'negritud', para Nacho, reside en una diferencia de estilo y de clase. La negritud tiene correlación, en este caso, según Nacho, con lo que denomina como "grasa": lo que no es "fino". Lo que no resiste un estándar refinado de costumbres tales como "hablar correctamente", "estar instruido", "vestirse mal" u "oler mal", "tener mal gusto".

Aunque no sea el caso concreto, en la relación que establece entre Albatros y Universitario, y la comparación con los habitantes de City Bell, sus caracterizaciones se van definiendo a medida que se presenta, dependiendo el contexto y, más aún, cuando cuenta quién es él en el espacio del rugby. Aunque no haya demasiadas diferencias estéticas entre los jugadores de Albatros o Universitario y los de La Plata Rugby Club (LPRC), Nacho insiste en agudizar la distinción. No es la negritud que sí amplía cuando adjetiva, negativamente, a algún joven que solemos cruzar en la calle, vestido con zapatillas deportivas, con un corte de pelo (desairado por Nacho, por los diferentes niveles en el largo del pelo: la conjunción de rapado y algunos centímetros más en el mismo corte, más el accesorio de una gorra), o el color de piel (percibida por Nacho como "negra"). Hemos compartido muchas charlas en la vereda de su gimnasio y él detenía la charla si algún joven con esas características estéticas pasaba cerca de nosotros, sugiriendo que "estamos cada vez peor".

Como tantos y tantas, Nacho porta con el estigma histórico edificado en Argentina sobre las clases populares: el hecho de asociar, como dice Adamovsky (2012), las condiciones económicas desfavorables con el color de la piel, confirmando el prejuicio que quien mantiene la relación de "piel más oscura" y habita en zonas menos urbanizadas, es "inferior" y poco apto para la "civilización". La "tez blanca" más las conductas morales y estéticas de la "gente del rugby", inhabilitan a pensar en la posibilidad de que el rugby fuera "cosa de negros". Esto nos deja ver que la estrategia discursiva que Nacho utiliza al compararse con los habitantes de City Bell, es circunstancial y que la "negritud" no existe. Excepto para estigmatizar y/o desjerarquizar a otros.

Nacho, oscila entre el relativismo que lo coloca a él y a su club en un lugar de privilegio, y un absolutismo que lo condena a él y a Albatros a la periferia del rugby platense; aunque jamás duda que el rugby sea, en La Plata y en Argentina, un espacio donde ciertos actores se distinguen y son distinguidos. Asegura fervientemente que el rugby no es un deporte popular, pero a la vez dice que cualquiera puede jugarlo, apoyándose en lo que el resto de mis interlocutores (cualquiera sea su club) sostiene: "Lo puede jugar cualquiera: el gordo, el flaco, el alto, el bajo. Todos”. Es una pluralidad fisonómica la que construyen Nacho y sus colegas. Y donde excluye a las mujeres, "porque tampoco es un deporte que la mujer entienda mucho, y como el deporte sigue siendo a ese nivel y a todo nivel, el rugby es un deporte lamentablemente elitista".

Nacho construye y lleva adelante una imagen que naturaliza en relación al ideal de jugador de rugby, en donde asocia la posición económica con la belleza como condición, estrictamente referida al rugby: gran volumen muscular (de cuellos, trapecios, brazos, pectorales, abdominales), corte de cabellos estándar (el límite del largo del cabello llega hasta por encima de los hombros. No registré jugador que

Ha sido un espacio importante de sociabilidad donde he conocido muchos aspectos sociales, culturales y estéticos del mundo del rugby. Todos los nombres que aparecen en este escrito son de ficción para resguardar la identidad.

10 Localidad vecina y de similares características a las de Gonnet, que conserva el prestigio al ser el barrio habitado por familias tradicionales de la ciudad de La Plata, o por contar entre sus moradores, con funcionarios con cargos jerárquicos en el gobierno de la Provincia de Buenos Aires, o empresarios influyentes en la órbita privada. City Bell, se ubica luego de Gonnet, trasladándose desde La Plata hacia Ciudad Autónoma de Buenos Aires (CABA). 
tuviera el pelo más largo de esa medida), rostros que considera de "tez blanca", y un buen aroma en la ropa y en el cuerpo. Y desde ahí, lo que esa articulación de atributos - supuestamente discriminados y complementados por Nacho- trae aparejado en el beneficio social que otorga en términos de estatus. Condición socioeconómica privilegiada, más un estándar de belleza dominante, enmarcados en la lógica elitista que organiza el rugby: el rugby sería para gente distinguida. Dice Nacho que él lo percibe como "aires de grandeza, aires de elitismo que genera el rugby".

Nacho moviliza, ante mí, y ante otros sujetos circunstanciales, (compañeros de rugby, mujeres, su pareja, su familia, clientes de su gimnasio) su identidad, que le sirve como factor de legitimidad de una posición, justamente de privilegio. Esa movilidad de clasificaciones, al decir de Ziegler y Gessaghi (2012), no son una descripción de la realidad -como construcción social-, sino una posición ante ella. Pensar en desnaturalizar la diferencia es pensar en reponer las relaciones históricas que Nacho expresa como naturaleza y esencia. Sobre todo, en la producción grupal de esos supuestos beneficios que Nacho caracteriza y distingue como resultado de "pertenecer" o "estar" en relaciones de privilegio, con respecto a otros grupos.

\section{Conclusiones}

Mediante este trabajo etnográfico y el cruce con la teoría sobre la clase social, se pudieron construir datos que permiten conceptualizar y cargar de significados a un deporte como el rugby, en Argentina.

Los círculos de sociabilidad vinculados al ocio (como el tiempo no necesariamente productivo y donde se puede encontrar algún tipo de regocijo en una trama de relaciones sociales), en el rugby, tendrán que ver con la construcción de la diferencia; la distinción de otros espacios moralmente no deseables. El rugby en La Plata será uno de los espacios de acceso y acumulación de prestigio, liderazgo y poder, instituyendo una de las bases para adquirir cierto estatus social, al decir de Joffre Dumazedier (1971), pensando los espacios de sociabilidad y ocio. En este caso, el ocio se vuelve posibilidad de establecer un valor potencial en la vida social de una ciudad y en la influencia que diferentes sujetos, a partir de sus estrategias, desarrollen entre esos círculos de acumulación de poder.

El rugby, desde la década de 1910 en delante, se cristalizará como el círculo de contención y tránsito de personajes que obtuvieron u obtendrán prestigio social. Será uno de los espacios donde se reproduzca esa cultura europea deseada por las bases de conformación de una nueva Nación. Será el espacio deportivo de distinguibilidad en la ciudad de La Plata y en Argentina. Será el lugar donde se perpetúe el sistema moral que distingue a los "caballeros" y a los "honrados hombres", cuyo prestigio social atribuido en la ciudad, se confirmará en la participación de un juego cargado de rudeza y agresión física. Es que también es el espacio donde se reproducirá el modelo masculino dominante por excelencia, según los criterios de clasificación de lo que, para el Estado, será un "verdadero hombre": templado, racional, culto, educado. Pero, complementariamente: viril, corajudo, audaz y valiente, con una hombría a sostener ante cualquier contingencia.

El rugby significa, como se ha repasado en la ciudad de La Plata, dentro de un contexto nacional, un espacio de atribución y conquista de un prestigio social reconocido entre círculos de privilegio. Es una escuela moral distintiva, donde se clasificó, históricamente, lo que significa ser un "verdadero hombre", a partir de un sistema de pautas dominantes y hegemónicas, emparentadas con el atributo de la heteronormatividad y con la exaltación de la virilidad, dentro y fuera del campo del rugby. El origen social y las trayectorias de los sujetos investigados demarcan las propiedades y la legitimidad propia y reconocida como principal para pertenecer al campo. El capital cultural adquirido, más una trayectoria ligada a una "buena familia distinguida" (reforzando la idea de los legados, como modo de reproducir y sostener el prestigio social), se emparentan no sólo a la posición compartida, en términos de clase, en relación a la posición en la estructura social y económica de los sujetos, sino también en la capacidad, destreza y poder de administrar las diferencias culturales y simbólicas. 
En síntesis, el rugby se convirtió en uno de los espacios para mantener y contribuir a la ilusión de estar cerca de Europa. En Buenos Aires, como en La Plata, se perpetraron y se conservan tradiciones, costumbres y valores relacionados con la cultura inglesa y francesa como posibilidad distintiva de "lo local". Es un giro hacia la distinguibilidad, directo y asociado a un privilegio autopercibido por los sujetos participantes del rugby. Es el espacio donde se enseñan las buenas y legítimas costumbres que, estratégica y eficazmente, construyen distinción moral, pero también estética, a la vez que se edifica una narrativa en donde la retórica del honor y la caballerosidad, diría Gayol (2008), proveen un lenguaje propio. Donde además de nombrar ese mundo como legítimo, se pone a prueba poniendo el cuerpo y exhibiéndolo, ya que el honor en el rugby, se asocia a la reputación social. Un prestigio que debe ser sostenido durante toda la estadía en el campo, si se pretende, verdaderamente, aspirar a ser distinguido social y culturalmente.

\section{Bibliografía}

Adamovsky, Ezequiel (2012), Historia de la clase media argentina. Buenos Aires: Grupo editorial Planeta, sexta edición.

Alabarces, Pablo (2006). El deporte en América Latina. En Enciclopedia Latinoamericana, Rio de Janeiro: CLACSO.

Alabarces, Pablo (2002). Fútbol y patria. Buenos Aires: Prometeo Ediciones.

Archetti, Eduardo (2001). El potrero, la pista y el ring. Las patrias del deporte argentino, Buenos Aires: FCE.

Bourdieu, Pierre (1998[1979]). La distinción. Criterios y bases sociales del gusto. Madrid: Editorial Taurus.

Branz, Juan y Garriga Zucal, José (2012). Civilizados y animales. Representaciones publicitarias de la identidad nacional en el rugby. Revista Oficios Terrestres "Comunicación y ciencias sociales en Latinoamérica". La Plata: Publicación de la Facultad de Periodismo y Comunicación Social, UNLP. $\mathrm{N}^{\circ} 27$.

Collins, Tony (2006). Rugby's Great Split. Class, Culture and the Origins of Rugby league Football [Gran partido division de Rugby. Clase, cultura y los orígenes de la Liga de Rugby Fútbol]. Londres: Routledge.

Dine, Philip (2007). Corps et genre: de la masculinité au rugby [Cuerpo y género: la masculinidad de rugby]. Corps 1/2007 (n 2), p. 37-41. <www.cairn.info/revue-corps-2007-1-page-37.htm>. En línea.

Dumazedier, Joffre y otros (1971). Ocio y sociedad de clases. Barcelona: Ed. Fontanella.

Gayol, Sandra (2008). Honor y duelo en la Argentina moderna. Buenos Aires: Siglo XXI Editores.

Gerth, Hans \& Wright Mills, Charles. (1963). Carácter y Estructura Social. Buenos Aires: Editorial Paidós.

Hall, Stuart (1994). Estudios Culturales: dos paradigmas. En Revista Causas y azares. ํㅜ 1. Buenos Aires.

Losada, Leandro (2012). La educación de la clase alta argentina. Vida doméstica e instituciones. En Ziegler, Sandra \& Gessaghi, Victoria (comp.), Formación de las elites. Investigaciones y debates en Argentina, Brasil y Francia. Buenos Aires: Ediciones Manantial.

Palermo, Elisa (2010). Deporte y clases sociales: notas sobre las significaciones sociales del rugby y el hockey en el Hurling Club. En II Seminario de discusión Investigación histórica y etnográfica sobre las clases medias en la Argentina, Buenos Aires: IDES.

Williams, Raymond (2003). Palabras clave. Un vocabulario de la cultura y la sociedad. Primera edición. Buenos Aires: Nueva Visión.

Ziegler, Sandra \& Gessaghi, Victoria (comp.) (2012). Formación de las elites. Investigaciones y debates en Argentina, Brasil y Francia. Buenos Aires: Ediciones Manantial. 